\title{
A proof of P != NP (New symmetric encryption algorithm against any linear attacks and differential attacks)
}

This paper was downloaded from TechRxiv (https://www.techrxiv.org).

\section{LICENSE}

CC BY 4.0

SUBMISSION DATE / POSTED DATE

22-02-2022 / 01-03-2022

\section{CITATION}

Ming, Gao (2022): A proof of P != NP (New symmetric encryption algorithm against any linear attacks and differential attacks). TechRxiv. Preprint. https://doi.org/10.36227/techrxiv.19212846.v1

$\mathrm{DOI}$ 


\title{
A proof of $\mathrm{P} !=$ NP (New symmetric encryption algorithm against any linear attacks and differential attacks)
}

\author{
Gao Ming \\ 20070602094@cqu.edu.cn
}

\section{Abstract}

P vs NP problem is the most important unresolved problem in the field of computational complexity. Its impact has penetrated into all aspects of algorithm design, especially in the field of cryptography. The security of cryptographic algorithms based on short keys depends on whether $\mathrm{P}$ is equal to NP. In fact, Shannon[1] strictly proved that the one-time-pad system meets unconditional security, but because the one-time-pad system requires the length of key to be at least the length of plaintext, how to transfer the key is a troublesome problem that restricts the use of the one-time-pad system in practice. Cryptography algorithms used in practice are all based on short key, and the security of the short key mechanism is ultimately based on "one-way" assumption, that is, it is assumed that a one-way function exists. In fact, the existence of one-way function can directly lead to the important conclusion $\mathrm{P} !=\mathrm{NP}$.

In this paper, we originally constructed a short-key block cipher algorithm. The core feature of this algorithm is that for any block, when a plaintext-ciphertext pair is known, any key in the key space can satisfy the plaintext-ciphertext pair, that is, for each block, the plaintext-ciphertext pair and the key are independence, and the independence between blocks is also easy to construct. This feature is completely 
different from all existing short-key cipher algorithms.

Based on the above feature, we construct a problem and theoretically prove that the problem satisfies the properties of one-way functions, thereby solving the problem of the existence of one-way functions, that is, directly proving that $P !=N P$.

\section{Introduction}

Cryptography is one of the most important applications in the field of communication and computer science. In recent years, with the application of commerce, enterprises, banks and other departments, cryptography has been developed rapidly. Especially after Shannon put forward the mathematical analysis of security in "Communication theory of secrecy systems" [1], various design tools for cipher algorithms and corresponding attack tools have been developed one after another. Among them, the most common attack methods include linear attacks and differential attack.

Linear attack was first proposed by M. Matsui [2], this is an attack method that is currently applicable to almost all block encryption algorithms. Kaliski BS [3] proposed a multi-linear attack based on the linear attack, but the multi-linear attack has many limitations. And the Biryukov A [4] and Chao, J.Y [5] and others further improved the framework of multi-linear attacks, thus making linear attacks a larger application.

The differential attack method was first proposed by Eli Biham [6]. BIHAM E [7] extended it to a more powerful attack method. TSUNOO [8] further constructed 
multiple attack methods. These attack methods have extremely high skill in the attack process, which is worthy in-depth study.

In this paper, we first designed a new encoding algorithm, which we named Eagle . The feature of the Eagle algorithm is that the output of the algorithm with the same input and the same parameters is completely random.

Based on the Eagle encoding algorithm, we designed a new block symmetric cipher algorithm. Based on this cipher algorithm, for any block of plaintext-ciphertext pairs, for any key in the key space, a suitable encryption method can be found. That is to say, there is no specific mathematical relationship between the plaintext, key, and ciphertext in each block, showing a completely randomly property. It can also be understood that for any plaintext, encrypted with the same key every time, the ciphertext obtained is not uniquely determined, but completely randomly in the possible ciphertext space. And this feature makes the cipher algorithm can resistant all forms of linear attacks and differential attacks.

At the end of this paper, we further construct a new cipher system. Under this cipher system, if any plaintext-ciphertext pair is known, if an attacker wants to guess the possible correct key, he cannot do it in polynomial time. We have proved theoretically that this kind of problem satisfies the properties of one-way functions, that is, theoretically prove that one-way functions exist, so that $P !=N P$.

\section{Introduction to Eagle encoding algorithm}

We first introduce two common bit operations. XOR denoted as $\oplus$. Do left cycle 
shift of $D$ by $n$ bits which can be denoted as $D^{+n}$, for example $(1001101)^{+2}=(0110110)$.

We select two $L$-bits parameters $w_{0}$ and $w_{1}$, have odd number of different bits. For example 11010011 and 10100101 have 5 bits (5 is odd) different.

\section{1 \\ 10100101 \\ $\mathrm{X} \times \mathrm{X} \times \mathrm{X}$}

We set the initial state of $L$-bit as $S_{0}$, we choose one parameter in $w_{0}$ or $w_{1}$, without loss of generality, assume that we choose $w_{0}$, then we define the following calculation

$S_{1}=w_{0} \oplus\left(S_{0} \oplus S_{0}^{+1}\right)$

From (3.1), we can easily know

$S_{0} \oplus S_{0}^{+1}=S_{1} \oplus w_{0}$

If we only know $S_{1}$, we don' t know whether $w_{0}$ or $w_{1}$ we used in (3.1), we can confirm it through a simple trial-and-error. For example, we guess the parameter $w_{1}$ was used in (3.1), we need to find a certain number $S_{x}$ to Satisfy

$S_{x} \oplus S_{x}^{+1}=S_{1} \oplus w_{1}$

In fact, since $w_{0}$ and $w_{1}$ have odd number of different bits, such $S_{x}$ does not exist. See Theorem 1 for details.

[Theorem 1] We arbitrarily choose two $L$-bit parameters $w_{0}$ and $w_{1}$ which have odd number of different bits, for arbitrary $S_{0}$, we set $S_{1}=w_{0} \oplus\left(S_{0} \oplus S_{0}^{+1}\right)$, then there doesn' t exists $S_{x}$ satisfy $S_{x} \oplus S_{x}^{+1}=S_{1} \oplus w_{1}$.

Proof: Firstly, by definition we get 
$S_{1} \oplus w_{1}=w_{0} \oplus\left(S_{0} \oplus S_{0}^{+1}\right) \oplus w_{1}=\left(S_{0} \oplus S_{0}^{+1}\right) \oplus\left(w_{0} \oplus w_{1}\right)$,

Where $w_{0}$ and $w_{1}$ have odd number of different bits, so there are odd number of 1 in the bit string of $w_{0} \oplus w_{1}$.

Suppose there have $S_{x}$ satisfy $S_{x} \oplus S_{x}^{+1}=S_{1} \oplus w_{1}$, then

$S_{x} \oplus S_{x}^{+1}=\left(S_{0} \oplus S_{0}^{+1}\right) \oplus\left(w_{0} \oplus w_{1}\right)$, by simple calculation we get

$\left(S_{0} \oplus S_{x}\right) \oplus\left(S_{0} \oplus S_{x}\right)^{+1}=w_{0} \oplus w_{1}$, we set $S_{y}=S_{0} \oplus S_{x}$, then there are odd number of 1 in the bit string of $S_{y} \oplus S_{y}^{+1}$, without of generality, we suppose that the bits with 1 are $l_{1}, l_{2}, \ldots, l_{u}$ ( $u$ is odd). Compare to the first bit of $S_{y}$, the $l_{1}+1$ bit of $S_{y}$ is different from the first bit of $S_{y}$, the $l_{2}+1$ bit of $S_{y}$ is the same with the first bit of $S_{y}$, the $l_{3}+1$ bit of $S_{y}$ is different from the first bit of $S_{y}$, the $l_{4}+1$ bit of $S_{y}$ is the same with the first bit of $S_{y}$, and so on, since $u$ is odd, the $l_{u}+1$ bit of $S_{y}$ is different from the first bit of $S_{y}$, by the definition of cycle shift, we conclude that the first bit of $S_{y}$ is different from the first bit of $S_{y}$, this is contradictory.

So there doesn' t exists $S_{x}$ satisfy $S_{x} \oplus S_{x}^{+1}=S_{1} \oplus w_{1}$.

Let's go back to the discussion just now, after a trial-and-error, we can accurately confirm which one ( $w_{0}$ or $\left.w_{1}\right)$ we just used in (3.1).

Now we suppose that there is a binary sequence $M=b_{1} b_{2} \ldots b_{L}$ with length $L$. Start with $S_{0}$, read each bit of $M$ from left to right sequentially, when the bit $b_{i}(1 \leq i \leq L)$ is 0 , we set $S_{i}=w_{0} \oplus\left(S_{i-1} \oplus{S_{i-1}}^{+1}\right)$, when the bit $b_{i}$ is 1 , we set $S_{i}=w_{1} \oplus\left(S_{i-1} \oplus S_{i-1}^{+1}\right)$.

According to the above calculation, for every $S_{i}$, we can find the only $w_{0}$ or $w_{1}$ 
such that there exists $S_{i-1}$ satisfy $S_{i-1} \oplus S_{i-1}^{+1}=S_{i} \oplus w_{x}$. According to the properties of XOR and cyclic shift, we can easily know that there are only two $S_{i-1}$ that satisfy $S_{i-1} \oplus S_{i-1}^{+1}=S_{i} \oplus w_{x}$, and the two $S_{i-1}$ that with each bit different. As long as we know any bit of $S_{i-1}, S_{i-1}$ can be uniquely determined. So we only need to save any bit of $S_{i}$, by $S_{L}$, we can completely restore the original state $S_{0}$ and the binary sequence $M$.

Based on the above discussion, we can construct a complete Eagle encoding and decoding algorithm. The entire algorithm consists of three processes: generating parameters, encoding, and decoding.

\section{[Parameter generation]}

Firstly we choose two $L$-bit parameters $w_{0}$ and $w_{1}$ which have odd number of different bits, then we choose $L$-bit initial state $S_{0}$.

\section{[Encoding]}

For input data $M$, we record $M[i](1 \leq i \leq L)$ as the $i$-th bit of $M, M[i] \in\{0,1\}$,

$L$ is the length of $M$, the encoding process is as follows.

[E1] Execute E2 to E4 with $i$ from 1 to $L$.

[E2] If $M[i]=0$, set $S_{i}=w_{0} \oplus\left(S_{i-1} \oplus S_{i-1}^{+1}\right)$.

[E3] If $M[i]=1$, set $S_{i}=w_{1} \oplus\left(S_{i-1} \oplus{S_{i-1}}^{+1}\right)$.

[E4] Set the last bit of $S_{i}$ as the $i$-th bit of $C, C[i]=S_{i}[L]$.

[E5] Use $\left(C, S_{L}\right)$ as the output.

\section{[Decoding]}

The output ( $C, S_{L}$ ) of the above encoding process is used as the input of the 
decoding process, the decoding process is as follows.

[D1] Execute D2 to D4 with $i$ from $L$ to 1.

[D2] Do trial-and-error testing with $S_{i} \oplus w_{0}$ or $S_{i} \oplus w_{1}$, find the unique $w_{x} \quad(x=0$ or $\quad x=1)$ satisfy $S_{x} \oplus S_{x}^{+1}=S_{i} \oplus w_{x}$.

[D3] After D2, use $x$ as the $i$-th bit of $M, M[i]=x$.

[D4] For the two possible $S_{x}$ satisfy $S_{x} \oplus S_{x}^{+1}=S_{i} \oplus w_{x}$ in D2, we set the one which the last bit is equal to $C[i]$ as $S_{i-1}$.

[D5] Use $M$ as the output.

It is not difficult to find that the above encoding process and decoding process are correct, that is $\left(C, S_{L}\right)$ generated by encoding from $M$ can be completely restored through the decoding process. In addition, the encoding process is sequential encoding in the order of $M^{\prime} \mathrm{s}$ bits, and the decoding process is sequential decoding in the reverse order of $C^{\prime} \mathrm{s}$ bits.

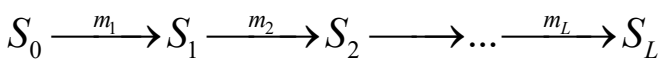

$S_{0} \longleftarrow c_{1} S_{1} \longleftarrow c_{2} S_{2} \longleftarrow \ldots \longleftarrow c_{L}-S_{L}$

We also noticed the fact that in the above encoding and decoding process, all inputs and outputs do not need to appear $S_{0}$, This means that the selection of $S_{0}$ will not affect the correctness of the encoding process and decoding process. The arbitrary of $S_{0}$ will bring the uncertainty of the encoded output.

For the convenience of the discussion in the following chapters, here we briefly analyze the effect of uncertainty of $S_{0}$ on the encoded output. Given the 
parameters $w_{0}$ and $w_{1}$ that have odd number of different bits, for a certain input $M$ of $L$ bits, since $S_{0}$ is arbitrarily selected, it is obvious that $C$ is uncertain, but is the final state $S_{L}$ necessarily uncertain?

In fact, the answer is no. In some cases, such as $L=2^{u}$ (that is, the parameter length is the power of 2), the final state $S_{L}$ is determined for different choices of $S_{0}$. The final state variable $S_{L}$ which is the output of the encoding process is only related to the input $M$ and has nothing to do with the choice of the initial state $S_{0}$. See Theorem 2 for details.

[Theorem 2] In Eagle encoding, given the parameters $w_{0}$ and $w_{1}$ that have $L$ bits with different odd bits, for a certain $L$ bit input $M$, if $L=2^{u}$ is satisfied, then for any initial state $S_{0}$, after the Eagle encoding process, the final state $S_{L}$ is only related to the input $M$, and is unrelated with the choice of the initial state $S_{0}$. Proof: We represent $M$ as binary stream $x_{1} x_{2} \ldots x_{L}$, which $x_{i} \in\{0,1\}, 1 \leq i \leq L$. We execute the Eagle encoding process to $M$ from $x_{1}$ to $x_{L}$ as follows.

$$
\begin{aligned}
& S_{1}=w_{x_{1}} \oplus\left(S_{0} \oplus S_{0}^{+1}\right)=f_{1}\left(w_{x_{1}}\right) \oplus\left(S_{0} \oplus S_{0}^{+1}\right) \\
& S_{2}=w_{x_{2}} \oplus\left(S_{1} \oplus S_{1}^{+1}\right)=f_{2}\left(w_{x_{1}}, w_{x_{2}}\right) \oplus\left(S_{0} \oplus S_{0}^{+2}\right) \\
& S_{3}=w_{x_{3}} \oplus\left(S_{2} \oplus S_{2}^{+1}\right)=f_{3}\left(w_{x_{1}}, w_{x_{2}}, w_{x_{3}}\right) \oplus\left(S_{0} \oplus S_{0}^{+1} \oplus S_{0}^{+2} \oplus S_{0}^{+3}\right) \\
& S_{4}=w_{x_{4}} \oplus\left(S_{3} \oplus S_{3}^{+1}\right)=f_{4}\left(w_{x_{1}}, w_{x_{2}}, w_{x_{3}}, w_{x_{4}}\right) \oplus\left(S_{0} \oplus S_{0}^{+4}\right)
\end{aligned}
$$

It is not difficult to find that for any $m=2^{v}, S_{m}=f_{m}\left(w_{x_{1}}, \ldots, w_{x_{m}}\right) \oplus\left(S_{0} \oplus S_{0}^{+m}\right)$ holds, this can be proved by a simple mathematical induction. In fact, the conclusion is correct for $v=1$. We assume that the conclusion is correct for $v-1$, we have $S_{m / 2}=f_{m / 2}\left(w_{x_{1}}, \ldots, w_{x_{m / 2}}\right) \oplus\left(S_{0} \oplus S_{0}^{+m / 2}\right)$, since $S_{m / 2} \quad$ to $S_{m}$ must do 
calculations with $m / 2$ steps, we have $S_{m}=f_{m}\left(w_{x_{1}}, \ldots, w_{x_{m}}\right) \oplus\left(S_{0} \oplus S_{0}^{+m / 2}\right) \oplus\left(S_{0}^{+m / 2} \oplus S_{0}^{+m}\right)=f_{m}\left(w_{x_{1}}, \ldots, w_{x_{m}}\right) \oplus\left(S_{0} \oplus S_{0}^{+m}\right)$

Since $L=2^{u}$, we have $S_{L}=f_{L}\left(w_{x_{1}}, \ldots, w_{x_{L}}\right) \oplus\left(S_{0} \oplus S_{0}^{+L}\right)$, where $f_{i}(\ldots)$ is irrelevant with $S_{0}$, by definition of cycle shift, we have $S_{0}=S_{0}^{+L}$, so $S_{L}=f_{L}\left(w_{x_{1}}, \ldots, w_{x_{L}}\right)$ is irrelevant with $S_{0}$.

From theorem 2, for any parameters $w_{0}$ and $w_{1}$ with length $L=2^{u}$, for any initial state $S_{0}$, execute Eagle encoding on $M$ to obtain $S_{L}$ which is irrelevant with $S_{0}$. In order to facilitate the description in the following chapters, we introduce the symbol $\xi$ and $\zeta$. $\xi_{w_{0}, w_{1}}:\left(S_{0}, M\right) \Rightarrow\left(S_{1}, C\right):$ use the key $w_{0}, w_{1}$ to execute Eagle encoding ([E1]-[E5]) on initial state $S_{0}$ and input $M$ to obtain $C$ and $S_{1}$. $\zeta_{w_{0}, w_{1}}:\left(S_{1}, C\right) \Rightarrow\left(S_{0}, M\right):$ use the key $w_{0}, w_{1}$ to execute Eagle decoding ([D1]-[D5]) on $C$ and $S_{1}$ to obtain $S_{0}$ and $M$.

In all the following chapters of this paper, we assume the length is a power of 2.

\section{Eagle encryption algorithm}

The core idea of Eagle encryption algorithm comes from the Eagle encoding process. If we use the parameters $w_{0}$ and $w_{1}$ in the Eagle encoding process as encryption keys, the process of encoding Input can be regarded as the process of encrypting plaintext Input. Output (Output, $S_{n}$ ) can be used as ciphertext. In fact, we can introduce uncertainty into the initial state $S_{0}$ without affecting the 
correctness of the decoding process. We will see later that uncertainty allows us to design a more secure encryption system.

Next, we will introduce the Eagle encryption algorithm in detail. The entire Eagle encryption algorithm is divided into three processes: key generator, encryption process, and decryption process.

\subsection{Eagle key generator}

First, the choice of the key is completely random, and the key needs to be shared between the encryptor and the decryptor. Since $w_{0}$ and $w_{1}$ must have odd number of different bits, there are only $2^{2 L-1}$ effective keys with bits length of $2 L$, one bit will be lost. That is, in the Eagle encryption algorithm, the number of bits for the key is always an odd number.

We randomly generate a number with bits length of $2 L$. We take the first $L$ bits as $w_{0}$. When the next $L$ bits are different from $w_{0}$ by an odd number of "bits", then we directly take the next $L$ bits as $w_{1}$; when the next $L$ bits are different from $w_{0}$ by an even number of "bits", we set the next $L$ bits as $w_{1}$ with the last bit inverted.

\subsection{Eagle encryption process}

For the $2 L-1$-bit key $w_{0}$ and $w_{1}$, for the plaintext $M$, we construct Eagle encryption processes as follows:

[M1] The plaintext $M$ is grouped by $L$ bits, and the last group with less than $L$ bits are randomly filled into $L$ bits. The total number of groups is assumed to be $T$, the grouped plaintext $M$ is recorded as $M_{1}, M_{2}, \ldots, M_{T}$. 
[M2] We randomly generate two numbers as the initial state $S_{0}$ and the random group $M_{T+1}$ inserted into $M_{1}, M_{2}, \ldots, M_{T}$.

[M3] Calculate from the first group to the $T+1$ group. For the first group $M_{1}$, Start state is $S_{0}$, after the encoding of [E1]-[E5], the state becomes $S_{1}$, and the encoded data is $C_{1}$ (Each bit of $C_{1}$ is composed of the last bit of all intermediate states), which are recorded as $\xi_{w_{0}, w_{1}}:\left(S_{0}, M_{1}\right) \Rightarrow\left(S_{1}, C_{1}\right)$. then we reset the state $S_{1}$ to $S_{1}=S_{1} \oplus C_{1}$. For the second group $M_{2}$ ， the state is $S_{1}$, after the encoding of [E1]-[E5], the state becomes $S_{2}$, and the encoded data is $C_{2}$, which are recorded as $\xi_{w_{0}, w_{1}}:\left(S_{1}, M_{2}\right) \Rightarrow\left(S_{2}, C_{2}\right)$, then reset $S_{2}=S_{2} \oplus C_{2}$. After $T+1$ groups, the final state is $S_{T+1}$, and the encoded data of each group is $C_{1}, C_{2}, \ldots, C_{T+1}$.

[M4] Output $C_{1} C_{2} \ldots C_{T+1} S_{T+1}$ as ciphertext.

\subsection{Eagle decryption process}

With the same key $w_{0}$ and $w_{1}$, for ciphertext $C_{1} C_{2} \ldots C_{T+1} S_{T+1}$, the Eagle decryption processes are as follows:

[C1] Starting from the last group $T+1$ to the first group, do decryption in sequence. For the $T+1$ group, we reset $S_{T+1}=S_{T+1} \oplus C_{T+1}$ and use $S_{T+1}$ and $C_{T+1}$ to execute the decoding operations [D1]-[D5] introduced in Chapter 2 to obtain state $S_{T}$ and decoded data $M_{T+1}$, which are recorded as $\zeta_{w_{0}, w_{1}}:\left(S_{T+1}, C_{T+1}\right) \Rightarrow\left(S_{T}, M_{T+1}\right)$. For the $T$ group, we reset $S_{T}=S_{T} \oplus C_{T}$ and use states $S_{T}$ and $C_{T}$ to execute the decoding operations [D1]-[D5] to obtain state $S_{T-1}$ and decoded data $M_{T}$, which are recorded as $\zeta_{w_{0}, w_{1}}:\left(S_{T}, C_{T}\right) \Rightarrow\left(S_{T-1}, M_{T}\right)$. After execute all the processes until the first group, the decoded data 
corresponding to each group is $M_{T+1}, M_{T}, \ldots, M_{1}$ respectively.

[C2] Output $M_{1} M_{2} \ldots M_{T}$ as the plaintext.

Obviously, the above decryption processes can get the correct plaintext.

In addition, for the plaintext with the $L$ bit, in the encryption or decryption process, it is encoded or decoded bit by bit, and the encoding or decoding process of each bit is a certain and direct calculation process, so the encryption process and the decryption process have computational complexity $O(L)$

\section{Linear attack analysis to Eagle encryption algorithm}

Linear attack is a very effective attack method against the DES algorithm proposed by M. Matsui [2] at the European Cryptographic Conference in 1993. Later, scholars quickly discovered that the linear attacks are applicable to almost all block encryption algorithms, and linear attacks have became the main attacks for block encryption algorithms. Various new attacks based on linear attacks are constantly being proposed.

The core idea of linear attack is to take the nonlinear transformation in the cryptographic algorithm, such as the linear approximation of the S-box, and then extend the linear approximation to the linear approximation of the round function, and then connect these linear approximations to obtain the entire cryptographic algorithm a linear approximation, and finally a large number of known plaintext-ciphertext pairs encrypted with the same key are used to exhaustively obtain the plaintext and even the key. 
We have noticed that the reason why linear attacks have become an effective attack for block encryption algorithms is that when the key is known, there is a certain implicit linear relationship between the ciphertext and the plaintext. By analyzing the known plaintext-ciphertext pairs, some effective linear relations can be obtained, and some bits of the key can be guessed.

In the Eagle encryption processes, for a certain group, suppose that the initial state at the beginning of the group is $S_{i-1}$, the plaintext of the group is $M_{i}$, the keys are $w_{0}$ and $w_{1}$, after the [E1]-[E5], we obtain the new state $S_{i}$ and the encoding result $C_{i}$. Only $C_{i}$ is included in the ciphertext, and $S_{i}$ is not included in the ciphertext, that is, $S_{i}$ is invisible to the decryption party and thus invisible to the attacker.

At the beginning of each group, $S_{i-1}$ can be regarded as completely random, this is because from the first group, $S_{0}$ is completely random, after the encoding process for the first group, $C_{1}$ is completely random, the state $S_{1} \oplus C_{1}$ of the second group is also completely random. By analogy, the initial state $S_{i-1}$ at the beginning of each group is completely random, and the ciphertext $C_{i}$ of the group is completely random.

That is to say, for a certain key and plaintext, the ciphertext is completely random, which have no mathematical relationship between the plaintext or the key. For an attacker, under the condition that a pair of plaintext $M_{i}$ and ciphertext $C_{i}$ of any group is known, for any key in the key space, a corresponding encryption or decryption method can be found to meet the conditions. For example, if an attacker chooses a specific key $w_{0}$ and $w_{1}$, he can choose $S_{i-1}^{\prime}$ randomly, and then 
use the key pair $S_{i-1}{ }^{\prime}$ and $M_{i}$ to execute the encoding process of [E1]-[E5] to get the state $S_{i}$. According to the conclusion of [Theorem 2], the arbitrariness of the attacker's choice of $S_{i-1}{ }^{\prime}$ does not affect the certainty of $S_{i}$. Then he can execute the decoding process of [D1]-[D5] according to $S_{i}$ and $C_{i}$ to get $S_{i-1}$. This means that by any key he can find a suitable $S_{i-1}$ that satisfies the condition for a known plaintext $M_{i}$-ciphertext $C_{i}$ pair, and the attacker cannot get any valid information from the pair, which can be denoted as $\operatorname{Pr}\left(W=\left(w_{0}, w_{1}\right) \mid\left(M=m_{i}, C=c_{i}\right)\right)=1 /\left(2^{2 L-1}\right)$. This can also be summarized as the following theorem:

[Theorem 3] For any $L$ bit $M_{1}$ and $C_{1}$, for any $2 L-1$ bit $K=\left(w_{0}, w_{1}\right)$, there must be unique $S_{0}$ and $S_{1}$, so that $\xi_{w_{0}, w_{1}}:\left(S_{0}, M_{1}\right) \Rightarrow\left(S_{1}, C_{1}\right)$ holds.

The independence of the plaintext-ciphertext pair with the key makes it impossible for any linear attacker to establish an effective relationship.

\section{Differential attack analysis to Eagle encryption algorithm}

Differential attack was proposed by Biham and Shamir [6] in 1990, it is a chosenplaintext attack. Its core idea is to obtain key information by analyzing specific plaintext and ciphertext differences.

The essence of a differential attack is to track the "difference" of the plaintext pair, where the "difference" is defined by the attacker according to the target, which can be an exclusive XOR operation or other target values. For example, if you choose the plaintext $M$ and the difference $\delta$, the other plaintext is $M+\delta$. The attacker 
mainly analyzes the possible keys by analyzing the difference between the ciphertext $C$ and $C+\varepsilon$.For the Eagle encryption algorithm, suppose the differential attacker chooses two specific plaintexts $M_{1}$ and $M_{2}$, their difference is $\delta$, that is $M_{2}=M_{1}+\delta$, the corresponding ciphertexts are $C_{1}$ and $C_{2}$, and the difference between the ciphertexts is $\varepsilon$, and That is $C_{2}=C_{1}+\varepsilon$. Since in the encryption processes of Eagle algorithm, $C_{1}$ and $C_{2}$ are completely random, it is completely uncertain whether the difference $\varepsilon$ of the ciphertext is caused by randomness or the spread of the plaintext. Furthermore, $E_{w_{0}, w_{1}}\left(M_{1}\right)$ and $E_{w_{0}, w_{1}}\left(M_{2}\right)$ subject to the same probability distribution, which can be denoted as $\operatorname{Pr}\left(C_{1}=c_{1}, C_{2}=c_{2} \mid\left(M_{1}=m_{1}, M_{2}=m_{2}\right)\right)=1 / 2^{2 L}$.

That is to say, for any specific plaintext $M_{1}$ and $M_{2}$ selected by the attacker, after being encrypted with the same key, the corresponding block ciphertexts $C_{1}$ and $C_{2}$ are completely random, and any possible value in the ciphertext space appears with equal probability. The attacker has no way to capture the propagation characteristics of the "difference" in the plaintext.

\section{One-way function design}

\subsection{Introduction to one-way functions}

Before constructing the one-way function, we briefly introduce the properties of one-way function and the relationships with the $\mathrm{P} !=$ NP problem.

[Definition 1] A function is a one-way function means that the function satisfies the following properties: 
a) For a given $x$, there exists a polynomial-time algorithm that output $f(x)$.

b) Given $y$, it is difficult to find an $x$ that satisfies $y=f(x)$, that is, there does not exists a polynomial-time algorithm that finding the $x$.

The NP-complete problem refers to a set of problems that are verifiable in polynomial-time algorithm. For all NP-complete problems, whether there exists algorithms that are solvable in polynomial-time, this is the $\mathrm{P}$ vs NP problem. If $\mathrm{P} !=$ NP, then for some NP problems, there is no algorithm that is solvable in polynomial-time. If $\mathrm{P}=\mathrm{NP}$, then for all NP problems, there exists algorithms that are solvable in polynomial-time.

If one-way function exists, it means that there exists such an NP problem, which has no deterministic polynomial time solvable algorithm, that is, $\mathrm{P} !=\mathrm{NP}$. This is a direct inference, which can be directly described as the following theorem, See [9] for details.

[Theorem 4] If one-way function exists, then P != NP.

We then introduce an additional simple algorithmic problem, which we describe as the following theorem.

[Theorem 5] For two sets selected completely independently, the number of elements is $l_{1}, l_{2}$, then the average algorithm complexity of finding the common elements of the two sets (there may be only one common element at most) is at least $c^{*} \min \left(O\left(l_{1}\right), O\left(l_{2}\right)\right)$, where $c$ is a certain constant.

This is because the remaining unvisited elements in the two sets will be visited at least once with equal probability before no common element is found. 


\subsection{Construction of one-way functions}

Let us go back to the Eagle encryption algorithm mentioned in Chapter 4. Assuming that the key is $K=\left(w_{0}, w_{1}\right)$, with length $2 L-1$ bit, the plaintext has $n$ blocks, denoted as $M=\left(M_{1}, M_{2}, \ldots, M_{n}\right)$ where each block with length $L$ bit, and the encrypted ciphertext is $C=\left(C_{1}, C_{2}, \ldots, C_{n}, C_{n+1}, S_{n+1}\right)$.

Among them, the plaintext-ciphertext pair of the $i(1 \leq i \leq n)$-th group is $M_{i}-C_{i}$, and the $n+1$ th group has only the ciphertext $\left(C_{n+1}, S_{n+1}\right)$ known.

It is not difficult to see that since the last group has only ciphertext, any key is "right".

For the $i(1 \leq i \leq n)$ group, we might take the first group without loss of generality. Given the plaintext $M_{1}$ and the ciphertext $C_{1}$, from Theorem 3, any key is also "right", so we can only "guess" the key from each group independently.

The plaintext-ciphertext pair between two discontinuous groups can also be regarded as completely independent, such as the plaintext or ciphertext between the $i$-th group and the $i+2$ th group has no relationship.

For two consecutive groups, the only connection between $M_{i}-C_{i}$ and $M_{i+1}-C_{i+1}$ lies in the initial state of the $i+1$-th group equal to $S_{i} \oplus C_{i}$.

In fact, we can easily cut off the connection between the $i$-th group and the $i+1$-th group. For example, change the initial state of the $i+1$-th group to $S_{i} \oplus Y=S_{i+1}$, where $Y$ is a random number that does not depend on any system variables. Then put $Y$ into the increased group to encrypt, so that when the plaintext-ciphertext pairs of any multiple groups are known, the process of 
"guessing" the key is independent for any grouping, and because Within each group, any key is "right", and the only way to "guess" the key is to use trial-and-error method.

Since the key is $2 L-1$ bits, there are $2^{2 L-1}$ possible keys in the entire key space. Obviously, the computational complexity of the trial-and-error method is exponential.

The above analysis is the core idea for us to construct a one-way function, and then we define the following encryption algorithm:

[Encryption Algorithm Q] For $2 L-1$ bit key $K=\left(w_{0}, w_{1}\right)$, for two blocks of plaintext $M=\left(M_{1}, M_{2}\right)$, the encryption process is as follows:

[Q1]. Generate $p \quad L$-bit random numbers completely independently, denoted as $X=\left(X_{1}, X_{2}, \ldots, X_{p}\right)$, where $p>1$.

[Q2]. Select three functions $f_{1}: x->y, f_{2}: x->y, f_{3}: x->y$, where $x$ is a set composed of $p L$-bit binary data, and $y_{1}, y_{2}, y_{3}$ are composed of $L$-bit binary data. Calculate $Y_{1}=f(X), Y_{2}=f_{2}(X), Y_{3}=f_{3}(X)$. Note that the selection of $f_{1}, f_{2}, f_{3}$ here are polynomial-time complexity.

[Q3]. Random numbers $S_{0}$ and $M_{3}$ are selected completely independently, and the following algorithm is executed:

$$
\begin{aligned}
& \xi_{Y_{2}, Y_{3}}:\left(S_{0}, M_{1}\right) \Rightarrow\left(S_{1}, C_{1}\right) \\
& \xi_{Y_{2}, Y_{3}}:\left(S_{1} \oplus Y_{1}, M_{2}\right) \Rightarrow\left(S_{2}, C_{2}\right) \\
& \xi_{Y_{2}, Y_{3}}:\left(S_{2}, M_{3}\right) \Rightarrow\left(S_{3}, C_{3}\right)
\end{aligned}
$$

Output $\left(C_{1}, C_{2}, C_{3}, S_{3}\right)$ as the first part of the ciphertext. 
[Q4]. Use the Eagle encryption algorithm introduced in Chapter 4 to encrypt $X=\left(X_{1}, X_{2}, \ldots, X_{p}\right) \quad$ to obtain the second part of the ciphertext $\left(C_{1}^{\prime}, C_{2}^{\prime}, \ldots, C_{p}^{\prime}, C_{p+1}^{\prime}, S_{p+1}^{\prime}\right)$.

Obviously, the algorithm Q is correct, because according to the ciphertext $\left(C_{1}, C_{2}, C_{3}, S_{3}\right)$ and $\left(C_{1}^{\prime}, C_{2}^{\prime}, \ldots, C_{p}^{\prime}, C_{p+1}^{\prime}, S_{p+1}^{\prime}\right)$, the plaintext $\left(M_{1}, M_{2}\right)$ can be completely decrypted.

First use the Eagle encryption algorithm introduced in Chapter 4 to decrypt $\left(C_{1}^{\prime}, C_{2}^{\prime}, \ldots, C_{p}^{\prime}, C_{p+1}^{\prime}, S_{p+1}^{\prime}\right) \quad$ to get $X=\left(X_{1}, X_{2}, \ldots, X_{p}\right)$, execute $Y_{1}=f_{1}(X)$, $Y_{2}=f_{2}(X), Y_{3}=f_{3}(X)$, and then execute the following algorithm:

$\zeta_{Y_{2}, Y_{3}}:\left(S_{3}, C_{3}\right) \Rightarrow\left(S_{2}, M_{3}\right)$

$\zeta_{Y_{2}, Y_{3}}:\left(S_{2} \oplus Y_{1}, C_{2}\right) \Rightarrow\left(S_{1}^{*}, M_{2}\right)$

$\zeta_{Y_{2}, Y_{3}}:\left(S_{1}^{*} \oplus Y_{1}, C_{1}\right) \Rightarrow\left(S_{0}, M_{1}\right)$

Output the complete plaintext $\left(M_{1}, M_{2}\right)$.

The question we are going to ask is that in algorithm $Q$, if we know the plaintext $\left(M_{1}, M_{2}\right)$, what is the benefit for us to "guess" the key $K=\left(w_{0}, w_{1}\right)$.

We divide the $\mathrm{Q}$ algorithm into two parts, [Q1]-[Q2] are the parameter preparation stages, [Q3] is the first part of the algorithm, and [Q4] is the second part of the algorithm. Obviously these two parts are completely independent. The only connection is $Y_{1}=f_{1}(X), Y_{2}=f_{2}(X), Y_{3}=f_{3}(X)$. Since $f_{1}, f_{2}, f_{3}$ can be regarded as compression functions, Unique $Y_{1}, Y_{2}, Y_{3}$ can be obtained for any $X$; for $Y_{1}, Y_{2}, Y_{3}$, there are $2^{(p-3) L-1} X$ satisfying $Y_{1}=f_{1}(X), Y_{2}=f_{2}(X), Y_{3}=f_{3}(X)$. From the decryption process, we can see that for the specified key $K=\left(w_{0}, w_{1}\right)$, a 
unique $X$ can be obtained, so that $Y_{1}, Y_{2}, Y_{3}$ are uniquely determined.

But without knowing the key $K=\left(w_{0}, w_{1}\right)$, the verification of $Y_{1}$ has also become a very "complex" problem.

This is because for the plaintext-ciphertext pair in the first part, for any $Y_{1}$, there are at least $2^{L-1}$ possible $Y_{2}, Y_{3}$ that are "right" .

For the ciphertext of the second stage, only $Y_{1}$ is known, even though $Y_{2}, Y_{3}$ are known, there are at least $2^{(p-3) L-1}$ possible $X$ that is "right" . So the problem of verifying $Y_{1}$ becomes an exponentially-time problem. Therefore, it can be directly proved that the problem of "guessing" the key satisfies the property of a one-way function. See the following theorem and proof for details.

[Theorem 6] For a $2 L-1$ bits key $K=\left(w_{0}, w_{1}\right)$, for two blocks of plaintext $M=\left(M_{1}, M_{2}\right)$, the ciphertext obtained after using the encryption algorithm $\mathrm{Q}$ are $\left(C_{1}, C_{2}, C_{3}, S_{3}\right)$ and $\left(C_{1}^{\prime}, C_{2}^{\prime}, \ldots, C_{p}^{\prime}, C_{p+1}^{\prime}, S_{p+1}^{\prime}\right)$, then under the condition that the plaintext $M=\left(M_{1}, M_{2}\right)$ is known, the problem of verifying $Y_{1}$ generated in [Q2] has no polynomial-time algorithms.

Proof: Observe the encryption algorithm $\mathrm{Q}$, there are five independent variables $K, X, Y_{1}, Y_{2}, Y_{3}$, [Q2] and [Q3] can be expressed as $F_{1}\left(X, Y_{1}, Y_{2}, Y_{3}\right)=0$, [Q4] expressed as $F_{2}(K, X)=0$.

Without knowing $K$ and $X$, the problem of verifying $Y_{1}=y_{1}$ is equivalent to the problem of finding $X$ that satisfy $F_{1}\left(X, Y_{1}, Y_{2}, Y_{3}\right)=0$ and $F_{2}(K, X)=0$.

For the part [Q3], given $M=\left(M_{1}, M_{2}\right)$ and $\left(C_{1}, C_{2}, C_{3}, S_{3}\right)$, by the following calculation process: 
$\xi_{Y_{2}, Y_{3}}:\left(S_{0}, M_{1}\right) \Rightarrow\left(S_{1}, C_{1}\right)$

$\xi_{Y_{2}, Y_{3}}:\left(S_{1} \oplus Y_{1}, M_{2}\right) \Rightarrow\left(S_{2}, C_{2}\right)$

With $C_{1}, C_{2}, M_{2}$ fixed, for any combination of $\left(Y_{2}, Y_{3}, Y_{1}\right)$, a unique $\left(S_{0}, M_{1}, S_{2}\right)$ can be derived. According to the simple combinatorics principle, it is not difficult to conclude that for any $y_{1} \in Y_{1}$, there are at least $2^{L-1}$ possible $y_{2}, y_{3} \in Y_{2}, Y_{3}$ that satisfy the condition. Therefore, by any algorithm, to verify $y_{1} \in Y_{1}$, it is necessary to verify each of the possible $2^{(p-2) l-1} X$.

For the part [Q4], under the condition that $\left(C_{1}^{\prime}, C_{2}^{\prime}, \ldots, C_{p}^{\prime}, C_{p+1}^{\prime}, S_{p+1}^{\prime}\right)$ is known, since $K$ is unknown, there are $2^{2 L-1}$ possible $X$.

Since the process [Q4] is independent from [Q2][Q3], by theorem 5, the problem of finding $X$ have work with exponent-complexity.

[Theorem 7] For a $2 L-1$-bits key $K=\left(w_{0}, w_{1}\right)$, for two blocks of plaintext $M=\left(M_{1}, M_{2}\right)$, the ciphertext obtained after using the encryption algorithm $\mathrm{Q}$ are $\left(C_{1}, C_{2}, C_{3}, S_{3}\right)$ and $\left(C_{1}^{\prime}, C_{2}^{\prime}, \ldots, C_{p}^{\prime}, C_{p+1}^{\prime}, S_{p+1}^{\prime}\right)$, then under the condition that the plaintext $M=\left(M_{1}, M_{2}\right)$ is known, The process of finding the key $K=\left(w_{0}, w_{1}\right)$ satisfies the properties of the one-way function.

Proof: First, for any key $K=\left(w_{0}, w_{1}\right)$, to determine whether it is correct, you only need to use $K$ to decrypt the ciphertext, and then determine whether the obtained plaintext is $M$. Obviously, the computational complexity is $O(L)$, which satisfies 7.1.1.

Next, we need to prove condition 7.1 .2 , that is, there is no polynomial-time algorithm for the problem of "guessing" the key. Here we adopt the method of 
proof by contradiction.

Assuming that there is a polynomial-time algorithm for the problem of "guessing the key", after the algorithm is executed, the ciphertext $\left(C_{1}, C_{2}, C_{3}, S_{3}\right)$ and $\left(C_{1}^{\prime}, C_{2}^{\prime}, \ldots, C_{p}^{\prime}, C_{p+1}^{\prime}, S_{p+1}^{\prime}\right)$ are decrypted to obtain $X$. The decryption only requires a complexity of $O(L)$. According to the assumption of [Q2], the calculation of $Y_{1}=f_{1}(X)$ is also polynomial-time complexity. And this series of calculations are all polynomial-time complexity, which contradicts the conclusion of Theorem 6.

Therefore, the problem of "guessing" the key satisfies the properties of one-way functions.

According to Theorem 7, we find a problem that satisfies the properties of one-way functions, which proves the existence of one-way functions. According to Theorem 4, we also directly proved $P$ != NP.

Finally, I will briefly introduce the selection of function $f_{i}(i=1,2,3)$ in [Q2]. In the process of proving that $f_{i}$ needs to satisfy the condition that for any $Y_{i}$, there are at least exponential possible $X$ satisfying $Y_{i}=f_{i}(X)$. This is actually very easy to do. For example, if we choose $p=L$, take the $i$-th bit of each number of $X_{1}, X_{2}, \ldots, X_{L}$ to satisfy the condition.

\section{[References]}

[1] Shannon C E . Communication theory of secrecy systems. 1945[J]. Bell System Technical Journal, 1949, 28(4):656 - 715. 
[2] M. Matsui. Linear cryptanalysis method for DES cipher[J]. Advances in cryptology: EUROCRYPT' 93, LNCS765, springer verlag, 1993:386-397.

[3] Kaliski B S , Robshaw M J B . Linear Cryptanalysis Using Multiple Approximations[C] Annual International Cryptology Conference. Springer, Berlin, Heidelberg, 1994.

[4] Biryukov, A., De Cannière, C., Quisquater, M.: On Multiple Linear Approximations. In: Franklin, M. (ed.) CRYPTO 2004. LNCS, vol. 3152, pp. 1-22. Springer, Heidelberg, 2004.

[5] Cho, J.Y., Hermelin, M., Nyberg, K.: A new technique for multidimensional linear cryptanalysis with applications on reduced round serpent. In: Lee, P.J., Cheon, J.H. (eds.) ICISC 2008. LNCS, vol. 5461, pp. 383-398. Springer, Heidelberg, 2009.

[6] Eli Biham, Adi Shamir. Differential Cryptanalysis of the Data Encryption Standard[M]. Springer-Verlag, 1993.

[7] BIHAM E, BIRYUKOV A, SHAMIR A. Cryptanalysis of Skipjack reduced to 31 rounds using impossible differentials[C]. In: Advances in Cryptology-EUROCRYPT 1999. Springer Berlin Heidelberg, 1999: 12-23.

[8] TSUNOO Y, TSUJIHARA E, SHIGERI M, et al. Cryptanalysis of CLEFIA using multiple impossible differentials[C]. In: 2008 International Symposium on Information Theory and Its Applications-ISITA 2008. IEEE, 2008: 1-6.

[9] Sanjeev Arora, Boaz Barak. Computational Complexity: A Modern Approach[J]. 2009. 